\title{
Selective Inhibition of Matrix Metalloproteinase Isozymes and in vivo Protection against Emphysema by Substituted $\gamma$-Keto Carboxylic Acids
}

Dawei Ma*, Yongwen Jiang, Fangping, Chen, Li-kun, Gong, Ke Ding, Yong Xu, Renxiao Wang, Aihua Ge, Jin Ren, Jingya Li, Jia Li, and Qizhuang Ye

State Key Laboratory of Bioorganic and Natural Products Chemistry, Shanghai Institute of Organic Chemistry, Chinese Academy of Sciences, 354 Fenglin Lu, Shanghai 200032, China, Shanghai Institute of Materia Medica, Chinese Academy of Sciences,555 Zuchongzhi Lu, Shanghai 201203, China, and Chinese National Center for Drug Screening, Shanghai Institute of Materia Medica, Shanghai Institute for Biological Sciences, Chinese Academy of Sciences, 189 Guo-Shou-Jing Road, Shanghai 201203, China

\section{Supporting information}

\section{Table of contents}

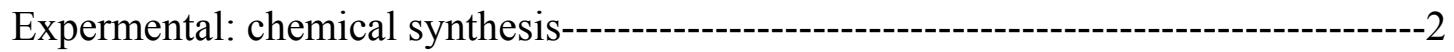

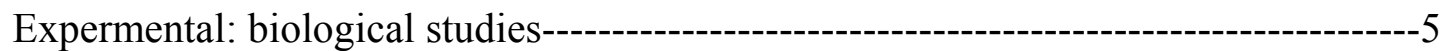

Purity of key compounds---_- 


\section{Expermental: chemical synthesis}

Alkylation of ketones 4 . To a solution of $4(0.9 \mathrm{mmol})$ in dry THF $(10 \mathrm{~mL})$ was added LDA (1.8 $\mathrm{M}$ in THF, $1.36 \mathrm{mmol})$ at $-78{ }^{\circ} \mathrm{C}$. The resultant solution was stirred for $1 \mathrm{~h}$ at -60 to $-78{ }^{\circ} \mathrm{C}$ before ethyl bromoacetate $(1.36 \mathrm{mmol})$ was added dropwise. After it was stirred at $-78{ }^{\circ} \mathrm{C}$ for $1 \mathrm{~h}$, the solution was allowed to warm to room temperature. The reaction mixture was partitioned between ethyl acetate and water. The organic layer was washed with $1 \mathrm{~N} \mathrm{HCl}$, saturated $\mathrm{NaHCO}_{3}$, and brine, respectively. Evaporation of the solution followed by column chromatography afforded alkylation products.

3-(4-Bromobenzoyl)nonanoic acid ethyl ester 4b. ${ }^{1} \mathrm{H}$ NMR $\left(\mathrm{CDCl}_{3}, 300\right.$ $\mathrm{MHz}) \delta 0.84(\mathrm{~m}, 3 \mathrm{H}), 1.26(\mathrm{~m}, 11 \mathrm{H}), 1.68(\mathrm{~m}, 2 \mathrm{H}), 2.53(\mathrm{dd}, J=4.8,16.8 \mathrm{~Hz}, 1 \mathrm{H})$, $2.92(\mathrm{dd}, J=9.9,16.8 \mathrm{~Hz}, 1 \mathrm{H}), 3.90(\mathrm{~m}, 1 \mathrm{H}), 4.10(\mathrm{q}, J=7.2 \mathrm{~Hz}, 2 \mathrm{H}), 7.62(\mathrm{~d}, J=$ $8.7 \mathrm{~Hz}, 2 \mathrm{H}), 7.87(\mathrm{~d}, J=8.7 \mathrm{~Hz}, 2 \mathrm{H})$, EI-MS $m / z 370\left(\mathrm{M}^{+},{ }^{81} \mathrm{Br}\right), 368\left(\mathrm{M}^{+},{ }^{79} \mathrm{Br}\right)$; HRMS calcd. for $\mathrm{C}_{18} \mathrm{H}_{25} \mathrm{BrO}_{3} 368.0985\left(\mathrm{M}^{+},{ }^{79} \mathrm{Br}\right)$, found 368.0993 .

4-(4-Bromophenyl)-3-cyclopentylmethyl-4-oxo-butyric acid ethyl ester 4c. ${ }^{1} \mathrm{H}$ NMR $\left(\mathrm{CDCl}_{3}, 300 \mathrm{MHz}\right) \delta 1.02(\mathrm{~m}, 2 \mathrm{H}), 1.15(\mathrm{t}, J=7.2 \mathrm{~Hz}, 3 \mathrm{H}), 1.40-1.79(\mathrm{~m}$, 9H), $2.56(\mathrm{dd}, J=4.5,16.8 \mathrm{~Hz}, 1 \mathrm{H}), 2.93(\mathrm{dd}, J=9.9,16.8 \mathrm{~Hz}, 1 \mathrm{H}), 3.85(\mathrm{~m}, 1 \mathrm{H})$, $4.06(\mathrm{q}, J=7.2 \mathrm{~Hz}, 2 \mathrm{H}), 7.60(\mathrm{~d}, J=8.7 \mathrm{~Hz}, 2 \mathrm{H}), 7.89(\mathrm{~d}, J=9.7 \mathrm{~Hz}, 2 \mathrm{H})$; EI-MS $m / z 368\left(\mathrm{M}^{+},{ }^{81} \mathrm{Br}\right), 366\left(\mathrm{M}^{+},{ }^{79} \mathrm{Br}\right)$; HRMS calcd. for $\mathrm{C}_{18} \mathrm{H}_{23} \mathrm{BrO}_{3} 366.0829\left(\mathrm{M}^{+},{ }^{79} \mathrm{Br}\right)$, found 368.00831 .

3-Benzyl-4-(4-bromophenyl)-4-oxo-butyric acid ethyl ester 4d. ${ }^{1} \mathrm{H}$ NMR $\left(\mathrm{CDCl}_{3}, 300 \mathrm{MHz}\right) \delta 1.14(\mathrm{t}, J=7.2 \mathrm{~Hz}, 3 \mathrm{H}), 2.49(\mathrm{dd}, J=4.2,16.8 \mathrm{~Hz}, 1 \mathrm{H}), 2.66$ 
$(\mathrm{dd}, J=8.4,13.5 \mathrm{~Hz}, 1 \mathrm{H}), 2.89-3.00(\mathrm{~m}, 2 \mathrm{H}), 4.00(\mathrm{q}, J=7.2 \mathrm{~Hz}, 2 \mathrm{H}), 4.10(\mathrm{~m}, 1 \mathrm{H})$, 7.12-7.32 (m, 5H), $7.59(\mathrm{~d}, J=8.4 \mathrm{~Hz}, 2 \mathrm{H}), 7.82(\mathrm{~d}, J=8.4 \mathrm{~Hz}, 2 \mathrm{H})$; EI-MS m/z 376 $\left(\mathrm{M}^{+},{ }^{81} \mathrm{Br}\right), 374\left(\mathrm{M}^{+},{ }^{79} \mathrm{Br}\right)$; HRMS calcd. for $\mathrm{C}_{19} \mathrm{H}_{19} \mathrm{BrO}_{3} 374.0516\left(\mathrm{M}^{+},{ }^{79} \mathrm{Br}\right)$, found 374.0518.

4-(4-Bromophenyl)-3-(4-fluorobenzyl)-4-oxo-butyric acid ethyl ester 4e. ${ }^{1} \mathrm{H}$ NMR $\left(\mathrm{CDCl}_{3}, 300 \mathrm{MHz}\right) \delta 1.16(\mathrm{t}, J=7.2 \mathrm{~Hz}, 3 \mathrm{H}), 2.43(\mathrm{dd}, J=4.8,17.1 \mathrm{~Hz}$, 1H), $2.65(\mathrm{dd}, J=7.8,13.8 \mathrm{~Hz}, 1 \mathrm{H}), 2.87-3.00(\mathrm{~m}, 2 \mathrm{H}), 4.04(\mathrm{q}, J=7.2 \mathrm{~Hz}, 2 \mathrm{H})$, $6.93(\mathrm{~m}, 2 \mathrm{H}), 7.07$ (m, 2H), $7.57(\mathrm{~d}, J=7.8 \mathrm{~Hz}, 2 \mathrm{H}), 7.78$ (d, $J=7.8 \mathrm{~Hz}, 2 \mathrm{H})$; EI-MS $m / z 394\left(\mathrm{M}^{+},{ }^{81} \mathrm{Br}\right), 392\left(\mathrm{M}^{+},{ }^{79} \mathrm{Br}\right)$; HRMS calcd. for $\mathrm{C}_{19} \mathrm{H}_{18} \mathrm{BrFO}_{3} 392.0422\left(\mathrm{M}^{+}\right.$, ${ }^{79} \mathrm{Br}$ ), found 392.0428 .

4-(4-Bromophenyl)-4-oxo-3-phenylbutyric acid ethyl ester 4f. ${ }^{1} \mathrm{H}$ NMR $\left(\mathrm{CDCl}_{3}, 300 \mathrm{MHz}\right) \delta 1.20(\mathrm{t}, J=7.2 \mathrm{~Hz}, 3 \mathrm{H}), 2.71(\mathrm{dd}, J=4.8,17.1 \mathrm{~Hz}, 1 \mathrm{H}), 3.34$ $(\mathrm{dd}, J=10.2,17.1 \mathrm{~Hz}, 1 \mathrm{H}), 4.11(\mathrm{q}, J=7.2 \mathrm{~Hz}, 2 \mathrm{H}), 5.00(\mathrm{dd}, J=4.8,10.2 \mathrm{~Hz}, 1 \mathrm{H})$, 7.19-7.29 (m, $5 \mathrm{H}), 7.53(\mathrm{~d}, J=8.7 \mathrm{~Hz}, 2 \mathrm{H}), 7.84(\mathrm{~d}, J=8.7 \mathrm{~Hz}, 2 \mathrm{H})$; EI-MS $m / z$ 362 $\left(\mathrm{M}^{+},{ }^{81} \mathrm{Br}\right), 360\left(\mathrm{M}^{+},{ }^{79} \mathrm{Br}\right)$; HRMS calcd. for $\mathrm{C}_{18} \mathrm{H}_{17} \mathrm{BrO}_{3} 360.0359\left(\mathrm{M}^{+},{ }^{79} \mathrm{Br}\right)$, found 360.0362 .

General procedure for preparation of $\gamma$-keto carboxylic acids 1 . To a solution of 4 (0.32 mmol), $\mathrm{PdCl}_{2}\left(\mathrm{PPh}_{3}\right)_{2} \quad(0.03 \mathrm{mmol}), \mathrm{CuI}$ (0.06 mmol), tetrabutylammonium iodide $(0.1 \mathrm{mmol})$ in $1 \mathrm{~mL}$ of DMF were added 1 -alkyne $(1.6$ mmol) and triethylamine $(0.5 \mathrm{~mL})$ via syringe under Ar atmosphere. The resultant mixture was heated at $75{ }^{\circ} \mathrm{C}$ until the aryl bromide was consumed monitored by TLC. The cooled solution was partitioned between ethyl acetate and water. The organic 
layer was washed with brine, and dried over $\mathrm{Na}_{2} \mathrm{SO}_{4}$. Evaporation of the solution followed by column chromatography afforded coupling products.

A mixture of the above $\gamma$-keto ester $(0.22 \mathrm{mmol}), 1 \mathrm{~mL}$ of $20 \%$ aqueous $\mathrm{NaOH}$, and $1 \mathrm{~mL}$ of methanol was stirred at room temperature. After it was acidified to $\mathrm{pH}=$ 1, the solution was extracted with ethyl acetate. The organic layers were washed with brine, and dried over $\mathrm{Na}_{2} \mathrm{SO}_{4}$. Evaporation of the solution followed by column chromatography afforded acids.

3-(4-Phenylethynylbenzoyl)nonanoic acid 1f. ${ }^{1} \mathrm{H} \mathrm{NMR}\left(\mathrm{CDCl}_{3}, 300 \mathrm{MHz}\right) \delta$ $0.86(\mathrm{t}, J=6.9 \mathrm{~Hz}, 3 \mathrm{H}), 1.22(\mathrm{~m}, 8 \mathrm{H}), 1.48(\mathrm{~m}, 1 \mathrm{H}), 1.67(\mathrm{~m}, 1 \mathrm{H}), 2.52(\mathrm{dd}, J=4.5$, $17.1 \mathrm{~Hz}, 1 \mathrm{H}), 3.00(\mathrm{dd}, J=9.0,17.1 \mathrm{~Hz}, 1 \mathrm{H}), 3.81(\mathrm{~m}, 1 \mathrm{H}), 7.36(\mathrm{~m}, 3 \mathrm{H}), 7.55(\mathrm{~m}$, 2H), $7.60(\mathrm{~d}, J=8.4 \mathrm{~Hz}, 2 \mathrm{H}), 7.93(\mathrm{~d}, J=8.4 \mathrm{~Hz}, 2 \mathrm{H})$; EI-MS $m / z 362\left(\mathrm{M}^{+}\right)$; HRMS calcd. for $\mathrm{C}_{24} \mathrm{H}_{26} \mathrm{O}_{3} 362.1880\left(\mathrm{M}^{+}\right)$, found 362.1887 .

3-Cyclopentylmethyl-4-oxo-4-((4-phenylethynyl)phenyl)butyric acid $1 \mathrm{~g}$. ${ }^{1} \mathrm{H}$ NMR $\left(\mathrm{CDCl}_{3}, 300 \mathrm{MHz}\right) \delta 1.01-1.10(\mathrm{~m}, 2 \mathrm{H}), 1.43-1.59(\mathrm{~m}, 5 \mathrm{H}), 1.62-1.82(\mathrm{~m}$, 4H), $2.60(\mathrm{dd}, J=4.2,17.4 \mathrm{~Hz}, 1 \mathrm{H}), 2.98(\mathrm{dd}, J=9.0,17.4 \mathrm{~Hz}, 1 \mathrm{H}), 3.88(\mathrm{~m}, 1 \mathrm{H})$, $7.37(\mathrm{~m}, 3 \mathrm{H}), 7.55(\mathrm{~m}, 2 \mathrm{H}), 7.60(\mathrm{~d}, J=8.1 \mathrm{~Hz}, 2 \mathrm{H}), 7.92(\mathrm{~d}, J=8.1 \mathrm{~Hz}, 2 \mathrm{H})$; EI-MS m/z $360\left(\mathrm{M}^{+}\right)$; HRMS calcd. for $\mathrm{C}_{24} \mathrm{H}_{24} \mathrm{O}_{3} 360.1723\left(\mathrm{M}^{+}\right)$, found 360.1729.

3-Benzyl-4-oxo-4-((4-phenylethynyl)phenyl)butyric acid 1 h. ${ }^{1} \mathrm{H}$ NMR $\left(\mathrm{CDCl}_{3}, 300 \mathrm{MHz}\right) \delta 2.46(\mathrm{dd}, J=4.5,17.4 \mathrm{~Hz}, 1 \mathrm{H}), 2.66(\mathrm{dd}, J=8.7,13.5 \mathrm{~Hz}, 1 \mathrm{H})$, $2.95(\mathrm{dd}, J=9.9,17.4 \mathrm{~Hz}, 1 \mathrm{H}), 3.05(\mathrm{dd}, J=5.4,13.5 \mathrm{~Hz}, 1 \mathrm{H}), 4.10(\mathrm{~m}, 1 \mathrm{H})$, 7.12-7.26 (m, $5 \mathrm{H}), 7.37$ (m, 3H), $7.54(\mathrm{~m}, 2 \mathrm{H}), 7.57$ (d, $J=8.4 \mathrm{~Hz}, 2 \mathrm{H}), 7.90$ (d, $J=$ 8.4 Hz, 2H); EI-MS m/z $368\left(\mathrm{M}^{+}\right)$; HRMS calcd. for $\mathrm{C}_{25} \mathrm{H}_{20} \mathrm{O}_{3} 368.1411\left(\mathrm{M}^{+}\right)$, found 
368.1413.

3-(4-Fluorobenzyl)-4-oxo-4-((4-phenylethynyl)phenyl)butyric acid 1i. ${ }^{1} \mathrm{H}$ NMR $\left(\mathrm{CDCl}_{3}, 300 \mathrm{MHz}\right) \delta 2.47(\mathrm{dd}, J=5.1,17.4 \mathrm{~Hz}, 1 \mathrm{H}), 2.70(\mathrm{dd}, J=8.4,13.8 \mathrm{~Hz}$, 1H), 2.93-3.07 (m, $2 \mathrm{H}), 4.08(\mathrm{~m}, 1 \mathrm{H}), 6.92(\mathrm{~m}, 2 \mathrm{H}), 7.09(\mathrm{~m}, 2 \mathrm{H}), 7.38(\mathrm{~m}, 3 \mathrm{H}), 7.54$ (m, 2H), $7.57(\mathrm{~d}, J=8.7 \mathrm{~Hz}, 2 \mathrm{H}), 7.91(\mathrm{~d}, J=8.7 \mathrm{~Hz}, 2 \mathrm{H})$; EI-MS $m / z 368\left(\mathrm{M}^{+}\right)$; HRMS calcd. for $\mathrm{C}_{25} \mathrm{H}_{19} \mathrm{FO}_{3} 386.1317\left(\mathrm{M}^{+}\right)$, found 386.1312 .

3-[4-(4-Chlorophenylethynyl)benzoyl]nonanoic acid 1j. ${ }^{1} \mathrm{H} \mathrm{NMR}\left(\mathrm{CDCl}_{3}\right.$, $300 \mathrm{MHz}) \delta 0.84(\mathrm{t}, J=6.6 \mathrm{~Hz}, 3 \mathrm{H}), 1.21-1.25(\mathrm{~m}, 8 \mathrm{H}), 1.50(\mathrm{~m}, 1 \mathrm{H}), 1.72(\mathrm{~m}, 1 \mathrm{H})$, $2.58(\mathrm{dd}, J=4.5,16.8 \mathrm{~Hz}, 1 \mathrm{H}), 3.00(\mathrm{dd}, J=9.6,16.8 \mathrm{~Hz}, 1 \mathrm{H}), 3.85(\mathrm{~m}, 1 \mathrm{H}), 7.36$ $(\mathrm{d}, J=8.7 \mathrm{~Hz}, 2 \mathrm{H}), 7.46(\mathrm{~d}, J=8.7 \mathrm{~Hz}, 2 \mathrm{H}), 7.62(\mathrm{~d}, J=7.8 \mathrm{~Hz}, 2 \mathrm{H}), 7.96(\mathrm{~d}, J=$ $7.8 \mathrm{~Hz}, 2 \mathrm{H})$; EI-MS m/z $396\left(\mathrm{M}^{+},{ }^{35} \mathrm{Cl}\right)$; HRMS calcd. for $\mathrm{C}_{24} \mathrm{H}_{25} \mathrm{ClO}_{3} 396.1490\left(\mathrm{M}^{+}\right.$, ${ }^{35} \mathrm{Cl}$ ), found 394.1493. Anal. Calcd for $\mathrm{C}_{24} \mathrm{H}_{25} \mathrm{ClO}_{3}$ : C: 72.63, $\mathrm{H}$ : 6.35; found: C: 72.54, H: 6.27.

\section{Expermental: biological studies}

Preparation of MMPs and inhibition assay. The encoded catalytic MMP sequence was amplified from the full-length gene and cloned into the pGEMEX-1 plasmid, individually. After sequence confirmation, the recombinant plasmid was transformed into the BL21 (DE3) strain for expression. The target catalytic domains of MMPs (MMP-1, 2, 7, 9, 12, 14, 15, 16 and 26) were purified and refolded as previous described. ${ }^{1-7}$

The typical inhibition assay was carried out in $100 \mu \mathrm{L}$ reaction system 
containing $50 \mathrm{mM}$ Tris-HCl, pH7.5, $10 \mathrm{mM} \mathrm{CaCl}_{2}, 10 \mu \mathrm{M} \mathrm{ZnCl}_{2}, 100 \mu \mathrm{M}$ TPL, 1 $\mathrm{mM}$ DTNB, 2\% DMSO and MMPs (5 $\mathrm{nM}$ for MMP-1, $10 \mathrm{nM}$ for MMP-2, $1 \mu \mathrm{M}$ for MMP-7, $15 \mathrm{nM}$ for MMP-9, $5 \mathrm{nM}$ for MMP-12, $300 \mathrm{nM}$ for MMP-14, $10 \mathrm{nM}$ for MMP-15, $10 \mathrm{nM}$ for MMP-16, $10 \mathrm{nM}$ for MMP-26) with different concentration of compounds. The enzymatic rate was recorded at $412 \mathrm{~nm}$ for $2 \mathrm{~min}$. Each measurement was done in triplicate to ensure statistically significant results. For comparison, a known potent MMP inhibitor, galardin was used for standard drug.

\section{Method of the pharmacological study of the compound $1 \mathrm{j}$ :}

Porcin pancreatic elastase-induced pulmonary emphysema in hamsters. Sixty male golden Syrian hamsters (110-120 g) divided into four equal groups. As previous described, ${ }^{8}$ emphysema was induced by single intratracheal injection with procine pancreatic elastse (PPE, $400 \mathrm{U} / \mathrm{kg}, 1 \mathrm{~mL} / \mathrm{kg}$ ) (Qianhong, Jiansu, China). The day of intratracheal injection with PPE or saline was designated day 0 . And the compound lj $5 \mathrm{mg} / \mathrm{kg} / \mathrm{d} \quad(2 \mathrm{~mL} / \mathrm{kg}$, dissolved in peanut oil) was injected intraperitoneally from day 1 to day 28 .

Stereology and histopathology examination. Hamsters were euthanized 1 and 28 days after PPE or saline injection, respectively. The heart was removed, and then the right ventricle and the left ventricle (include the interventricular septum) were separated and weighed. The lung was removed and the lung volume $\left(\mathrm{V}_{\mathrm{L}}\right)$ was measured using a plethysmometer, then the left lung was weighed. Relative lung volumes $\left(\mathrm{LV}, \mathrm{LV}=\mathrm{VL} /\right.$ body weight $\left.\times 10^{2}\right)$, and the index of right ventricular hypertrophy $(\mathrm{RVH}, \mathrm{RVH}=$ right ventricle/ (left ventricle + interventricular septum $) \mathrm{x}$ 
$\left.10^{3}\right)^{9}$ were calculated.

The right lung tissues were fixed in $4 \%$ phosphate-buffered paraformaldehyde ( $\mathrm{pH}$ 7.4) and processed for routine paraffin embedding. Serial sections $(5 \mu \mathrm{M})$ were cut and stained with hematoxylin and eosin (HE) and Weigert staining to assess the degree of emphysema. Alveolar mean linear intercept $(L \mathrm{~m})^{10}$ and area of elastic fibers were blindly detected quantitatively using LEICA QWin software of image analysis computer program (Leica Imaging Systems Ltd., Cambridge, England) as lung structural parameters of emphysema.

Statistical methods. Group mean values and standard deviations were calculated. After homogeneitic analysis, homogeneous data were analyzed with one-way analysis of variance with a post hoc test of least significant difference (LSD) to determine inter-group differences. Heterogeneous data were analyzed using nonparametric test. A value of $p<0.05$ was considered significant.

\section{References}

1. Lowry, C. L.; McGeehan, G.; LeVine, H. Proteins 1992, 12, 42.

2. Ye, Q. Z.; Johnson, L. L.; Yu, A.; Hupe, D. Biochemistry, 1995, 34, 4702.

3. Ye, Q. Z.; Johnson, L. L.; Baragi, V. Biochem. Biophys. Res. Commun. 1992, 186, 143.

4. Kroger, M.; Tschesche, H. Gene 1997, 196, 175.

5. Shimada, T.; Nakamura, H.; Ohuchi, E.; Fujii, Y.; Murakami, Y.; Sato, H.; Seiki, M.; Okada, Y. Eur. J. Biochem. 1999, 262, 90. 
6. Cheng, D. H.; Shen, Q.; Nan, F. J.; Qian, Z.; Ye, Q. Z. Protein Express. Purific. 2003, 27, 63 .

7. Ye, Q. Z.; Nan, F. J.; Hu, L. Y.; Qian, Z.; Qian, J. Acta Pharmacol. Sin. 2000, 21, 1021.

8. Lucey, E. C.; Goldstein, R. H.; Stone, P. J.; Snider, G. L. Am. J. Respir. Crit. Care Med. 1998, 158, 555.

9. Avelar, E.; Jalili, T.; Dong, L.; Arvizo, J.; Hu, P.; Litwin, S. E.; Mattson, J. P. BMC Physiology 2005, 5, No 6.

10. Ofulue, A. F.; Ko, M.; Abboud, R. T. Am. J. Physiol. 1998, 275 (Lung Cell. Mol. Physiol. 19), L1134-L1144. 
Purity of key compounds

\begin{tabular}{|c|c|}
\hline compounds & Analytical data \\
\hline 1f & $\begin{array}{l}\text { 92.8\% purity via HPLC (column: Ultimate XB-C18, } 150 \times 4.6 \\
\mathrm{~mm}, 5 \mathrm{mM} \text {, eluting with } 3: 7 \text { to } 1: 0(\mathrm{v} / \mathrm{v}) \text { of } 0.1 \% \text { TFA in } \\
\text { MeCN/0.1\% TFA in water) } \\
\text { 92.5\% purity via HPLC (column: Diamonsil XB-C18, } 150 \times 4.6 \\
\mathrm{~mm}, 5 \mathrm{mM} \text {, eluting with } 35: 65 \text { to } 1: 0(\mathrm{v} / \mathrm{v}) \text { of } 0.1 \% \text { TFA in } \\
\text { MeCN/0.1\% TFA in water) }\end{array}$ \\
\hline $1 g$ & $\begin{array}{l}\text { 96.4\% purity via HPLC (column: Ultimate XB-C18, } 150 \times 4.6 \\
\mathrm{~mm}, 5 \mathrm{mM} \text {, eluting with } 3: 7 \text { to } 1: 0(\mathrm{v} / \mathrm{v}) \text { of } 0.1 \% \mathrm{TFA} \text { in } \\
\text { MeCN/0.1\% TFA in water) } \\
\text { 95.7\% purity via HPLC (column: Diamonsil XB-C18, } 150 \times 4.6 \\
\mathrm{~mm}, 5 \mathrm{mM} \text {, eluting with } 35: 65 \text { to } 1: 0(\mathrm{v} / \mathrm{v}) \text { of } 0.1 \% \text { TFA in } \\
\text { MeCN/0.1\% TFA in water) }\end{array}$ \\
\hline $1 \mathbf{i}$ & $\begin{array}{l}\text { 97.9\% purity via HPLC (column: Ultimate XB-C18, } 150 \times 4.6 \\
\mathrm{~mm}, 5 \mathrm{mM} \text {, eluting with } 3: 7 \text { to } 1: 0(\mathrm{v} / \mathrm{v}) \text { of } 0.1 \% \text { TFA in } \\
\text { MeCN/0.1\% TFA in water) } \\
97.3 \% \text { purity via HPLC (column: Diamonsil XB-C } 18,150 \times 4.6 \\
\mathrm{~mm}, 5 \mathrm{mM} \text {, eluting with } 35: 65 \text { to } 1: 0(\mathrm{v} / \mathrm{v}) \text { of } 0.1 \% \text { TFA in } \\
\mathrm{MeCN} / 0.1 \% \text { TFA in water) }\end{array}$ \\
\hline $\mathbf{1 j}$ & $\begin{array}{c}\text { EA found } \mathrm{C}: 72.54, \mathrm{H}: 6.27 \\
\left(\text { Calcd for } \mathrm{C}_{24} \mathrm{H}_{25} \mathrm{ClO}_{3}: \mathrm{C}: 72.63, \mathrm{H}: 6.35\right)\end{array}$ \\
\hline
\end{tabular}

